\title{
Herida por proyectil de arma de fuego en cuello con lesión esofágica y migración intestinal
}

\author{
Edgar Meza-Félix,凶 Ricardo Balanzá-López, Santiago Moreno-Ordaz, Víctor Flores-Huerta, \\ Juan Carlos-Vázquez, Minero
}

Servicio de Cirugía General, Hospital Central de la Cruz Roja Mexicana, Ciudad de México.

Trabajo recibido: 24-I-2017; aceptado: 14-II-2017

\begin{abstract}
RESUMEN. En las grandes ciudades las lesiones por arma de fuego en la actualidad son comunes, muchas de ellas causan la muerte de los pacientes y otras dejan lesiones que ameritan manejo quirúrgico temprano. Algunas otras pueden tener lesiones o trayectos poco comunes. Presentamos el caso de una mujer de 27 años que ingresó al Servicio de Urgencias de la Cruz Roja Mexicana por herida por arma de fuego en hombro izquierdo sin salida. Al realizar estudios radiológicos simples (lateral de cervicales y AP de tórax) no se observó proyectil en cuello y tórax, observándose en abdomen. Se realizó tomografía donde lo único que se observa es aire prevertebral a nivel de C-7, en la exploración quirúrgica se apreció lesión en esófago cervical, la cual se cierra de manera primaria. Se realiza laparotomía encontrando proyectil en estómago. Las lesiones esofágicas por arma de fuego son poco comunes, por lo cual hay que tener un alto índice de sospecha para poder diagnosticarlas y darles manejo adecuado.
\end{abstract}

Palabras clave: Lesión esofágica, lesiones por arma de fuego.

ABSTRACT. Penetrating gunshot injuries are very common in big cities, lots of them causing death of patients and some more that require early surgical management. Some others may have uncommon injuries or uncommon path of the bullet. Introducing the case report of a 27 year old women, arriving the emergency department at Mexican Red Cross Hospital, Mexico City, with penetrating neck injury by gunshot on left shoulder with no exit hole, $X$ rays were realized (lateral cervical Rx and AP thorax Rx) with no evidence of bullet neither neck or thorax, with evidence in abdominal Rx, CT is realized and paravertebral air is evidenced at C7 level, surgical exploration it's done in which a cervical esophagus injury is observed, which is treated with primary closure, trauma exploratory laparotomy is performed and the bullet is found in stomach. Esophageal injuries by gunshot are uncommon and may need a high level of suspicion for early diagnosis and management.

Key words: Esophageal injury, gun shot injury.

\section{INTRODUCCIÓN}

Las heridas penetrantes en cuello son comunes en trauma en población civil, contando con un alto riesgo de lesiones significativas en cuello a estructuras vitales. Dependiendo del tipo de objeto que ocasione la lesión, en las heridas por arma de fuego, hasta el $50 \%$ de las víctimas presenta heridas significativas a diferencia de lesiones por instrumento cortante, en las cuales es del 10 a $20 \%{ }^{1}$

Las lesiones traumáticas del esófago se asocian con una alta mortalidad, la cual excede el $20 \%$, si existe perforación, la mortalidad puede elevarse hasta el $50 \%$ debido a infección pleural y mediastinal que llevará a sepsis y falla orgánica múltiple. Las características de la pared del esófago, su poca irrigación y la falta de adventicia hacen que el mismo sea muy susceptible para su perforación. ${ }^{2}$
La evaluación temprana de la lesión es vital, pues la mortalidad de lesiones inadvertidas supera el $90 \%$, la tomografía axial computarizada es el método más preciso para valoración paraesofágica de manifestaciones, la evaluación también puede incluir radiografía lateral de columna cervical, esofagograma con contraste hidrosoluble, laringoscopia y endoscopia flexible. ${ }^{2}$

En el trauma por proyectil de arma de fuego, el examen físico exhaustivo y el análisis clínico orientan al cirujano sobre los posibles órganos lesionados, de acuerdo con las condiciones clínicas del paciente y a la trayectoria del proyectil, la cual puede ser inferida, indirectamente, mediante el trazo establecido entre los orificios de entrada y de salida (en el caso de orificios pares). En los casos de orificio de entrada único, o de orificios impares, en pacientes clínicamente estables, los estudios radiológicos contribuyen, entre otras co- 
sas, a orientar al cirujano sobre dicha trayectoria. ${ }^{3} \mathrm{La}$ balística terminal estudia los efectos producidos por el proyectil de arma de fuego dentro del cuerpo de la víctima del trauma. Generalmente los proyectiles que comprometen varias regiones de la anatomía (cervical, torácica, abdominal o pélvica) o que cruzan la línea media, implican lesiones de mayor complejidad en cuanto a su tratamiento se refiere. ${ }^{3}$

Ante el hallazgo clínico de un orificio de entrada en la región cervical y la evidencia radiológica del proyectil alojado en el lado contralateral del abdomen, el equipo quirúrgico está en la obligación de:

1. Establecer la trayectoria del proyectil, descartando el posible compromiso de los órganos de las regiones anatómicas involucradas, en este caso, cuello, tórax y abdomen.

2. Diagnosticar de manera expedita las lesiones producidas.

3. Trazar la estrategia terapéutica. ${ }^{3}$

El trauma de esófago cervical generalmente es manejado quirúrgicamente. El esófago cervical es abordado mediante una incisión a lo largo del borde medial izquierdo del esternocleidomastoideo, el esófago debe ser expuesto y examinado de manera circunferencial, se sugiere realizar endoscopia intraoperatoria, de cualquier manera los principios de la reparación incluyen debridación de tejido necrótico o contaminado, cierre primario en las primeras 24 horas de evolución, siempre y cuando exista una adecuada vitalidad de tejido así como cierre sin tensión, realizando un cierre en uno o dos planos. ${ }^{4}$

Posterior a la reparación se recomiendan ayuno vía oral en lesiones de alto grado hasta realizar esofagograma con evidencia de adecuada reparación, y se puede realizar inicio temprano con dieta líquida en lesiones de bajo grado, se recomienda colocación de drenaje, aunque no está totalmente documentado. Si existe contaminación es muy recomendable para la prevención de formación de abscesos. ${ }^{4}$

\section{CASO CLÍNICO}

Se trata de una mujer de 27 años de edad que llegó con antecedente de herida por arma de fuego 30 minutos previos a su ingreso con dos heridas, una en cara y la otra en cuello. Se presenta consciente, orientada, con vía aérea permeable, sin datos de dificultad respiratoria, hemodinámicamente estable y refiriendo dolor intenso en cuello. TA 120/70 mmHg, FC 80 lpm, FR 21 rpm. Presentaba sangrado por cavidad oral, orificio de entrada en región maxilar izquierda y en región supraescapular izquierda, no se observaron orificios de salida. Tórax y abdomen sin datos patológicos. Se solicitó una radiografía lateral de columna cervical en donde se observó el primer proyectil alojado en seno maxilar izquierdo. En una radiografía de tórax no existieron datos patológicos ni evidencia del segundo proyectil.

Una radiografía de abdomen mostró el segundo proyectil en cuadrante superior derecho (figura 1). Se decidió tomar una TAC de cuello, tórax y abdomen, ya que el paciente se encontraba hemodinámicamente estable y debido a que los estudios previamente solicitados no eran concluyentes. Se observó fractura de $\mathrm{C} 7$ a nivel del tubérculo anterior, con aire en fascia prevertebral (figura 2) y el segundo proyectil en cavidad

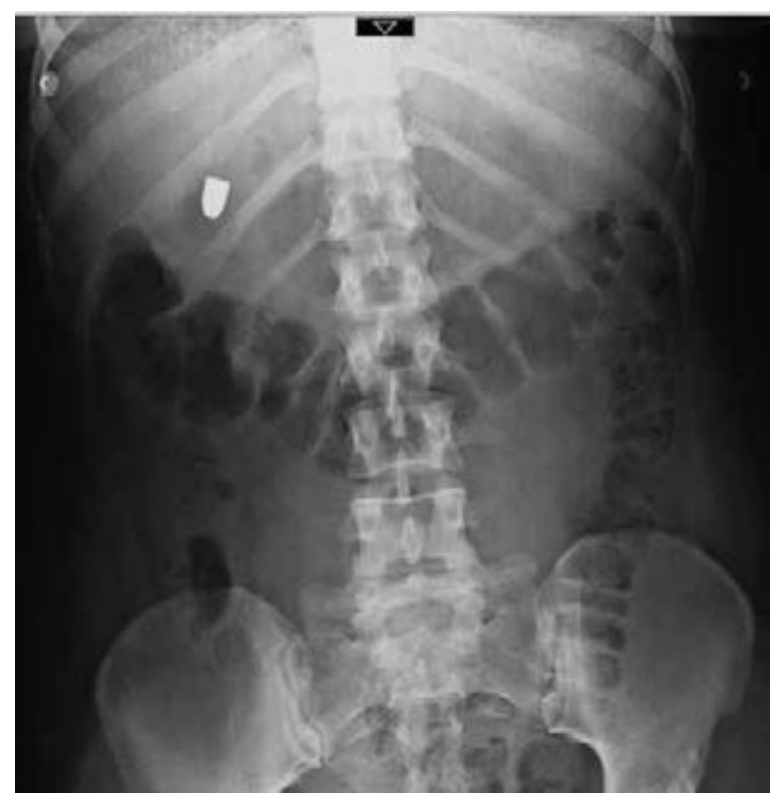

Figura 1. Proyectil en el abdomen.

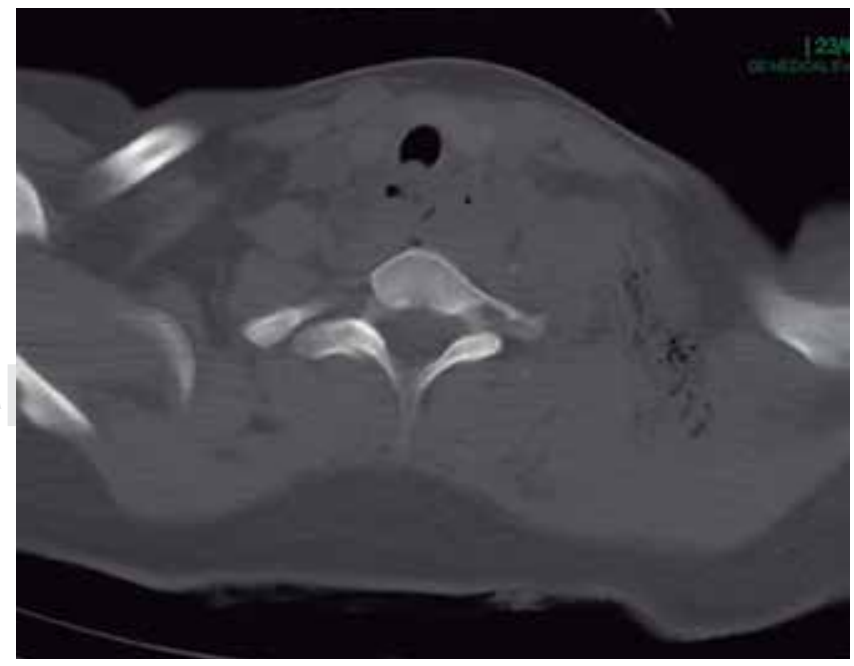

Figura 2. Tomografía de cuello con aire prevertebral. 
abdominal en topografía de estómago con artefacto, no se observó evidencia de líquido o aire libre. Se realizó un esofagograma en el que se identificó fuga de material hidrosoluble a nivel del esófago cervical, durante la realización de este estudio la paciente escupió el proyectil de la cara siendo éste de calibre $9 \mathrm{~mm}$ (figura 3). Se realizó exploración cervical identificando lesión grado III en pared lateral izquierda del esófago tratada con rafia con material absorbible multifilamento 3-0 y colocación de drenaje (figura 4). En la laparotomía exploradora no terapéutica se identificó el segundo proyectil intragástrico, deduciendo con ello que el proyectil fue «deglutido» y pasó a través del esófago al estómago. La paciente
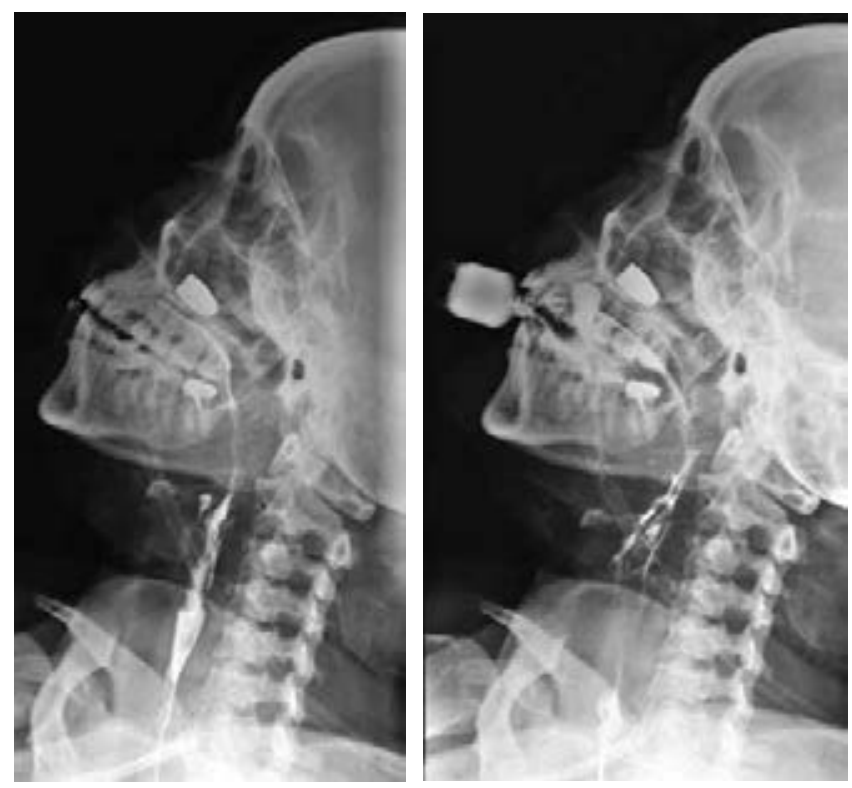

Figura 3. Esofagograma con fuga de contraste.

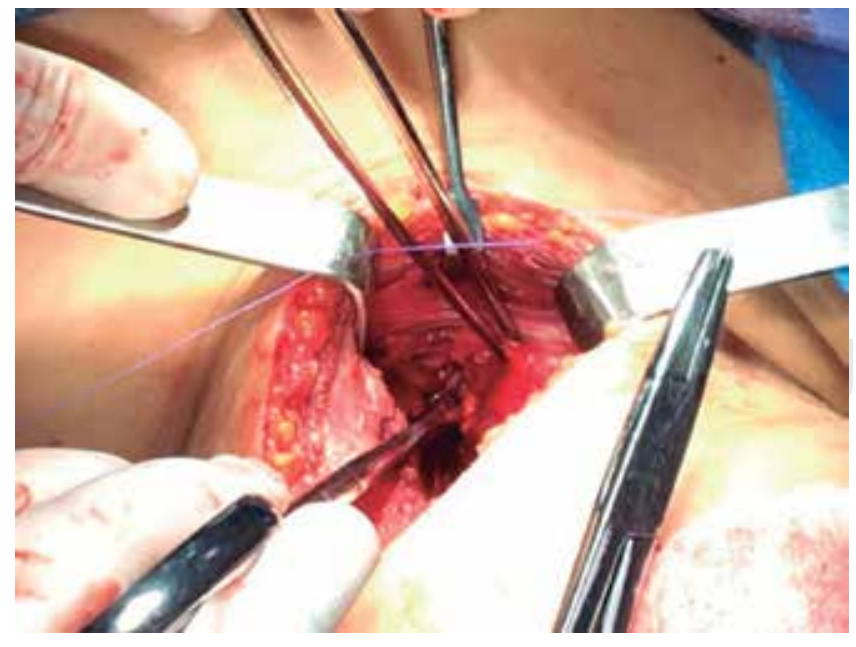

Figura 4. Lesión esofágica y reparación. curso su estancia intrahospitalaria con protección cervical, ayuno y soporte nutricional periférico por siete días. La paciente evacuó el proyectil en el quinto día posoperatorio siendo también calibre $9 \mathrm{~mm}$. Al séptimo día posoperatorio se realizó un nuevo esofagograma donde no se observó fuga del material de contraste, se inició vía oral, se retiró el drenaje al día siguiente y se dio de alta sin complicaciones con seguimiento al mes de operada con adecuada evolución.

\section{DISCUSIÓN}

El cuello es una región anatómica extremadamente compleja ya que múltiples estructuras vitales confluyen en un espacio pequeño. ${ }^{5,6}$ El riesgo de lesión por trauma penetrante depende del objeto utilizado, para heridas por arma de fuego es del $50 \%$, mientras que para heridas por instrumento punzocortante es del 10 al $20 \%$. ${ }^{1}$

En nuestro caso, el presentar mecanismo por arma de fuego, no tener salida y estar en la zona II, hacía obligatoria su revisión exhaustiva. En lesiones vasculares importantes la mortalidad supera el 50\%; en nuestra paciente el no tener lesión vascular acompañante mejoró su pronóstico., ${ }^{1,2,7} \mathrm{El}$ tratamiento clásico de heridas penetrantes de cuello dicta exploración quirúrgica para todos los pacientes con lesión en zona II; sin embargo, produce un 53 a $56 \%$ de exploraciones quirúrgicas negativas, por lo que deben realizarse estudios previos en pacientes estables como en esta paciente. .,7-9 $^{-1}$

La exploración física aislada es poco confiable en caso de lesiones esofágicas, pues el $80 \%$ puede ser negativa, en el caso presentado sólo existían los datos de dolor cervical intenso, sin ningún dato de compromiso esofágico. ${ }^{4}$ El manejo conservador es factible en pacientes estables con el apoyo de estudios de imagen, obviando el manejo quirúrgico hasta en un $82 \%$ de los casos, la conducta que tomamos en nuestra paciente al estar estable y no tener compromiso hemodinámico ni de vías aéreas fue el de realizar estudios complementarios., 1,5,7,9

La combinación de exploración física y estudios de imagen tiene una sensibilidad de $95 \%$ y un valor predictivo negativo de $97 \%$ para identificar lesiones que requieran manejo quirúrgico, gracias al dolor. El probable trayecto anómalo del proyectil de cuello a abdomen y los estudios con aire prevertebral hicieron que se sospechara en lesión del tracto digestivo., ${ }^{1,9}$

La presencia de enfisema subcutáneo tiene una sensibilidad de $94.1 \%$ y especificidad de $71.9 \%$ para identificar lesiones aerodigestivas, en nuestra paciente no existió a pesar de la lesión del esófago, sólo discreto aire a nivel prevertebral, lo que nos hizo sospechar de lesión digestiva y realizar más estudios. ${ }^{6}$ La angioTAC es el estudio de elección para descartar lesiones vas- 
culares, con una sensibilidad del 90 a $100 \%$ y especificidad del 98.6 al $100 \%$, pero también puede ayudar a sospechar otras lesiones al observar aire prevertebral muy lejano al de la entrada del proyectil y deducir una lesión esofágica., ${ }^{1,25-9}$

En caso de sospecha de lesión esofágica los estudios de elección son esofagograma o endoscopia. En nuestro centro hospitalario no contamos con endoscopia, por lo que se realizó el trago de medio de contraste obteniendo así el diagnóstico. ${ }^{1,4,7,8}$ Se recomienda utilizar medio de contraste hidrosoluble ya que no causa fibrosis mediastinal; sin embargo, puede existir un falso negativo hasta en un $22 \%$, por lo que al obtener un resultado negativo debe utilizarse bario para confirmar el resultado, nosotros lo realizamos con medio hidrosoluble. ${ }^{4}$

La endoscopia tiene una sensibilidad del $100 \%$ y especificidad del $92.4 \%$ para detectar lesiones esofágicas, nosotros no pudimos hacerlo. ${ }^{2}$

El manejo quirúrgico inmediato está indicado para pacientes con inestabilidad hemodinámica o con signos duros como hemorragia activa, hematoma expansivo 0 pulsátil, déficit de pulso, enfisema subcutáneo, estridor, compromiso de la vía aérea y hematemesis. ${ }^{1,5,7,8}$

En caso de lesión esofágica por arma de fuego en donde no se identifica un orificio de salida se ha descrito la migración del proyectil a través del tracto gastrointestinal por un mecanismo de «deglución» y su posterior evacuación. Lo interesante del caso que presentamos es que los estudios no mostraban un trayecto a través de estructuras como la pared torácica, pulmón o diafragma; más bien, nos hacía sospechar que el proyectil había sido deglutido y así pasó desde el esófago cervical hasta el estómago. ${ }^{3,10}$

El abordaje de elección para el esófago cervical es a través de una incisión en el borde medial del músculo esternocleidomastoideo izquierdo o bilateral, el abordaje que realizamos fue paralelo al esternocleidomastoideo. ${ }^{4,7}$ Los principios de la reparación esofágica incluyen escisión de tejido necrótico o contaminado, cierre del defecto y drenaje. El cierre primario está indicado en lesiones de menos de 24 horas de evolución con posibilidad de afrontar tejido sano sin tensión. No se debe iniciar vía oral hasta realizar un esofagograma y documentar el cierre adecuado de la lesión. En caso de lesiones destructivas está indicado realizar una esofagostomía. Nuestro cierre fue antes de las seis horas de la lesión con material absorbible. Se inició dieta hasta siete días después y posterior al estudio contrastado. ${ }^{4}$

\section{CONCLUSIÓN}

Este caso ejemplifica que el sospechar las lesiones que pueden causar las heridas por arma de fuego, hace que las busquemos de manera intencionada con recursos paraclínicos y no dejar solamente a la evolución clínica estos pacientes.

\section{REFERENCIAS}

1. Tisherman SA, Bokhari F, Collier B, et al. Clinical practice guideline: penetrating zone II neck trauma. J Trauma 2008;64(5):1392-1405. doi: 10.1097/ TA.0b013e3181692116.

2. Makhani M, Midani D, Goldberg A, Friedenberg FK. Pathogenesis and outcomes of traumatic injuries of the esophagus. Dis Esophagus 2014;27(7):30-636. doi: 10.1111/dote.12132.

3. Pereira GF, Romero BC, Arcia A, Suárez O. «Proyectil deglutido»: Ilustración de una trayectoria raramente observada en trauma cervical pediátrico por arma de fuego. Cir Pediatr 2007;20(4):229-231.

4. BiffI WL, Moore EE, Feliciano DV, et al. Western Trauma Association Critical Decisions in Trauma: Diagnosis and management of esophageal injuries. J Trauma Acute Care Surg 2015;79(6):1089-1095. doi: 10.1097/ TA.0000000000000772.

5. Prichayudh S, Choadrachata-anun J, Sriussadaporn S, et al. Selective management of penetrating neck injuries using "no zone" approach. Injury 2015;46(9):1720-1725. doi: 10.1016/j.injury.2015.06.019.

6. Madsen AS, Oosthuizen G, Laing GL, Bruce JL, Clarke $\mathrm{DL}$. The role of computed tomography angiography in the detection of aerodigestive tract injury following penetrating neck injury. J Surg Res 2016;205(2):490-498. doi: 10.1016/j.jss.2016.06.044.

7. Sperry JL, Moore EE, Coimbra R, et al. Western Trauma Association critical decisions in trauma: penetrating neck trauma. J Trauma Acute Care Surg 2013;75(6):936940. doi: 10.1097/TA.0b013e31829e20e3.

8. Siau RT, Moore A, Ahmed T, Lee MS, Tostevin P. Management of penetrating neck injuries at a London trauma centre. Eur Arch Otorhinolaryngol 2013;270(7):2123-2128. doi: 10.1007/s00405-0122324-9.

9. Van Waes OJ, Cheriex KC, Navsaria PH, van Riet PA, Nicol AJ, Vermeulen J. Management of penetrating neck injuries. Br J Surg 2012;99 Suppl 1:149-154. doi: 10.1002/bjs.7733.

10. Morrow JS, Haycock CE, Lazaro E. The "swallowed bullet" syndrome. J Trauma 1978;18(6):464-466.

\section{$\bowtie$ Correspondencia:}

Dr. Edgar Meza Félix

Servicio de Cirugía General, Hospital Central de la

Cruz Roja Mexicana, Ciudad de México.

Ejército Nacional Núm. 1032, colonia Polanco, C.P. 11510,

Miguel Hidalgo, Ciudad de México.

Tel: 53951111, ext. 104 o 214

Correo electrónico: edgar.meza230189@gmail.com

Los autores declaran no tener conflicto de intereses. 